Georgian Mathematical Journal

Volume 14 (2007), Number 4, 711-719

\title{
ELUSIVE EXAMPLES OF NON-METRIZABLE CONTINUA WHICH ADMIT A WHITNEY MAP
}

\author{
IVAN LONČAR
}

\begin{abstract}
The main purpose of this paper is to prove that the class of near locally connected continua contains no non-metrizable continuum $X$ which admits a Whitney map for $C(X)$. In particular, each near locally connected continuum $X$ which admits a Whitney map for $C(X)$ is metrizable.
\end{abstract}

2000 Mathematics Subject Classification: 54F15, 54C30.

Key words and phrases: Metrizability, Whitney map.

\section{INTRODUCTION}

The concept of Whitney maps is a very powerful tool in hyperspace theory of metric compact spaces. It is very natural to consider this concept in the settings of Hausdorff compact spaces. Unfortunately, in this case the existence of the Whitney maps for $2^{X}$ is equivalent to the metrizability of $X[1$, Theorem 1, pp. 305-306]. It remains to consider the Whitney maps for the hyperspace $C(X)$ since there exists a non-metrizable continuum which admits a Whitney map for $C(X)$ [1, p. 306]. However, one can observe that in the literature there is only one example of such a continuum. It would be desirable to construct such examples but we have not been able to do this. We will prove that in some families of continua there are no such examples. A very large class of such continua is the class of D-continua [7]. We will show that there exists a non D-continuum which is metrizable if and only if it admits a Whitney map. The main Theorems 3.2, 3.4 and 3.6 show why it is difficult to find non-metrizable continua $X$ for which there exists a Whitney map for $C(X)$.

All spaces in this paper are compact Hausdorff and all mappings are continuous. The weight of a space $X$ is denoted by $\mathrm{w}(X)$. The cardinality of a set $A$ is denoted by $\operatorname{card}(A)$.

A generalized arc is a Hausdorff continuum with exactly two non-separating points (end points) $x, y$. Each separable arc is homeomorphic to the closed interval $I=[0,1]$.

We say that a space $X$ is arcwise connected if for every pair $x, y$ of points of $X$ there exists a generalized arc $L$ with end points $x, y$.

Let $X$ be a space. We define its hyperspaces as the following sets:

$$
\begin{aligned}
& 2^{X}=\{F \subseteq X: F \text { is closed and non-empty }\}, \\
& C(X)=\left\{F \in 2^{X}: F \text { is connected }\right\} \\
& C^{2}(X)=C(C(X)), \\
& X(n)=\left\{F \in 2^{X}: F \text { has at most } n \text { points }\right\}, n \in \mathbb{N} .
\end{aligned}
$$


For any finitely many subsets $S_{1}, \ldots, S_{n}$, let

$$
\left\langle S_{1}, \ldots, S_{n}\right\rangle=\left\{F \in 2^{X}: F \subset \bigcup_{i=1}^{n} S_{i}, \text { and } F \cap S_{i} \neq \varnothing, \text { for each } i\right\} \text {. }
$$

The topology on $2^{X}$ is the Vietoris topology, i.e., the topology with a base $\left\{<U_{1}, \ldots, U_{n}>\right.$ : $U_{i}$ is an open subset of $X$ for each $i$ and each $\left.n<\infty\right\}$, and $C(X), X(n)$ are subspaces of $2^{X}$. Moreover, $X(1)$ is homeomorphic to $X$.

Let $X$ and $Y$ be two spaces and let $f: X \rightarrow Y$ be a mapping. Define $2^{f}: 2^{X} \rightarrow 2^{Y}$ by $2^{f}(F)=f(F)$ for $F \in 2^{X}$. By [9, Theorem 5.10, p. 170] $2^{f}$ is continuous and $2^{f}(C(X)) \subset C(Y), 2^{f}(X(n)) \subset Y$. The restriction $2^{f} \mid C(X)$ is denoted by $C(f)$.

Let $\Lambda$ be a subspace of $2^{X}$. By a Whitney map for $\Lambda$ [10, (0.50), p. 24] we will mean any mapping $g: \Lambda \rightarrow[0,+\infty)$ satisfying the conditions

a) if $A, B \in \Lambda$ such that $A \subset B$ and $A \neq B$, then $g(A)<g(B)$ and

b) $g(\{x\})=0$ for each $x \in X$ such that $\{x\} \in \Lambda$.

If $X$ is a metric continuum, then there exists a Whitney map for $2^{X}$ and $C(X)$ ([10, pp. 24-26], [3, p. 106]). On the other hand, if $X$ is non-metrizable, then it admits no Whitney map for $2^{X}[1]$. It is known that there exist non-metrizable continua which admit and ones which do not admit a Whitney map for $C(X)$ [1, p. 306].

The notion of an irreducible mapping was introduced by Whyburn [13, p. 162]. If $X$ is a continuum, a surjection $f: X \rightarrow Y$ is irreducible provided no proper subcontinuum of $X$ maps onto all of $Y$ under $f$. Some theorems for the case where $X$ is semi-locally-connected are given in [13, p. 163].

A mapping $f: X \rightarrow Y$ is said to be hereditarily irreducible [10, (1.212.3), p. $204]$ provided that for any given subcontinuum $Z$ of $X$, no proper subcontinuum of $Z$ maps onto $f(Z)$.

A mapping $f: X \rightarrow Y$ is light (zero-dimensional) if all fibers $f^{-1}(y)$ are hereditarily disconnected (zero-dimensional or empty) [2, p. 450], i.e., if $f^{-1}(y)$ does not contain any connected subset of cardinality larger that one ( $\left.\operatorname{dim} f^{-1}(y) \leq 0\right)$. Every zero-dimensional mapping is light, and in the realm of mappings with compact fibers the two classes of mappings coincide. Every hereditarily irreducible mapping is light. If $f: X \rightarrow Y$ is monotone and hereditarily irreducible, then $f$ is one-to-one.

Proposition 1 ([10, (1.212.3), p. 204]). A mapping $f: X \rightarrow Y$ is hereditarily irreducible if and only if the mapping $C(f): C(X) \rightarrow C(Y)$ is light.

We will use the notion of a inverse system as in [2, pp. 135-142]. An inverse system is denoted by $\mathbf{X}=\left\{X_{a}, p_{a b}, A\right\}$ and its limit is denoted by $\lim \mathbf{X}$.

We say that an inverse system $\mathbf{X}=\left\{X_{a}, p_{a b}, A\right\}$ is $\sigma$-directed if for each sequence $a_{1}, a_{2}, \ldots, a_{k}, \ldots$ of the members of $A$ there is $a \in A$ such that $a \geq a_{k}$ for each $k \in \mathbb{N}$.

Let $\mathbf{X}=\left\{X_{a}, p_{a b}, A\right\}$ be an inverse system of compact spaces with natural projections $p_{a}: \lim \mathbf{X} \rightarrow X_{a}$, for $a \in A$. Then $2^{\mathbf{X}}=\left\{2^{X_{a}}, 2^{p_{a b}}, A\right\}, C(\mathbf{X})=$ $\left\{C\left(X_{a}\right), C\left(p_{a b}\right), A\right\}$ and $\mathbf{X}(n)=\left\{X_{a}(n), 2^{p_{a b}} \mid X_{b}(n), A\right\}$ form inverse systems. 
Lemma 1.1. Let $X=\lim \mathbf{X}$. Then $2^{X}=\lim 2^{\mathbf{X}}, C(X)=\lim C(\mathbf{X})$ and $X(n)=\lim \mathbf{X}(n)$.

The following theorem is an external characterization of non-metrizable continua which admit a Whitney map for $C(X)$ [6, Theorem 2.3, p. 398].

Theorem 1.2. Let $X$ be a non-metrizable continuum. Then $X$ admits a Whitney map for $C(X)$ if and only if for each $\sigma$-directed inverse system $\mathbf{X}=$ $\left\{X_{a}, p_{a b}, A\right\}$ of continua which admit Whitney maps for $C\left(X_{a}\right)$ and $X=\lim \mathbf{X}$ there exists a cofinal subset $B \subset A$ such that for every $b \in B$ the projection $p_{b}: \lim \mathbf{X} \rightarrow X_{b}$ is hereditarily irreducible.

In the sequel we will use the following result [11, Exercise 11.52, p. 226].

Lemma 1.3. If $X$ is a continuum and if $A$ and $B$ are mutually disjoint subcontinua of $X$, then there is a component $K$ of $X \backslash(A \cup B)$ such that $\mathrm{Cl}(K) \cap$ $A \neq \varnothing$ and $\mathrm{Cl}(K) \cap B \neq \varnothing$.

We will use the notion of a network of a topological space.

A family $\mathcal{N}=\left\{M_{s}: s \in S\right\}$ of a subsets of a topological space $X$ is a network for $X$ if for every point $x \in X$ and any neighbourhood $U$ of $x$ there exists $s \in S$ such that $x \in M_{s} \subset U[2$, p. 170]. The network weight of a space $X$ is defined as the smallest cardinal number of the form $\operatorname{card}(\mathcal{N})$, where $\mathcal{N}$ is a network for $X$; this cardinal number is denoted by nw $(X)$.

The following theorem is due to Arkhangelski (see [2, Theorem 3.1.19, pp. $171,576])$.

Theorem 1.4. For every compact space $X$ we have $\mathrm{nw}(X)=\mathrm{w}(X)$.

\section{Hyperspace $C_{\text {int }}(X)$}

Now we will consider the subspace $C_{\text {int }}(X)$ of $C(X) \backslash X(1)$ containing all subcontinua of $X$ with non-empty interior. It is clear that $C_{i n t}(X)$ is non-empty since $X \in C_{\text {int }}(X)$.

Lemma 2.1. The hyperspace $C_{\text {int }}(X)$ is arcwise connected.

Proof. Let $K \in C_{\text {int }}(X)$. There exists an order arc $\alpha$ from $K$ to $X \in C(X)[10$, Theorem (1.12), p. 65] or [3, Theorem 14.9, p. 113]. It is clear that each $L \in \alpha$ has a non-empty interior (in $X$ ) since $K \subset L$ and $K$ has a non-empty interior in $X$. Thus, $\alpha \subset C_{\text {int }}(X)$.

It is a question whether $C_{\text {int }}(X)=C(X) \backslash X(1)$. We say that a continuum $X$ is completely regular if each non-degenerate subcontinuum of $X$ has a nonempty interior in $X[12$, p. 45]. Let us note that each completely regular continuum is the inverse limit of an inverse system of graphs with monotone bonding surjections [12, Corollary 7.14, p. 54]. Each completely regular continuum is hereditarily locally connected.

Lemma 2.2. If $X$ is a continuum, then $C_{\text {int }}(X)=C(X) \backslash X(1)$ if and only if $X$ is completely regular. 
Theorem 2.3. If $X$ is a continuum which admits a Whitney map for $C(X)$, then $C_{\text {int }}(X)$ is a separable metric space.

Proof. If $X$ is metrizable, then $C(X)$ is metrizable and separable since $\mathrm{w}(X)=$ $\mathrm{w}\left(2^{X}\right)=\aleph_{0}\left[2\right.$, Problem 3.12.26 (a), p. 306]. Hence, $C_{\text {int }}(X)$ is metrizable and separable.

Suppose that $X$ is non-metrizable. The remaining part of the proof is devided into several steps.

Step 1. There exists a $\sigma$-directed directed inverse system $\mathbf{X}=\left\{X_{a}, p_{a b}\right.$, $A$ \} of metric compact spaces $X_{a}$ such that $X$ is homeomorphic to $\lim \mathbf{X}[6$, Theorem 1.8, p. 397].

Step 2. There exists a cofinal subset $B \subset A$ such that for every $b \in B$ the projection $p_{b}: \lim \mathbf{X} \rightarrow X_{b}$ is hereditarily irreducible. This follows from Theorem 1.2.

Step 3. For every pair $C, D$ of disjoint non-degenerate subcontinua of $\lim \mathbf{X}$ with non-empty interiors there exists a non-degenerate subcontinuum $E \subset \lim \mathbf{X}$ such that $C \cap E \neq \varnothing \neq D \cap E$ and $(C \cup D) \backslash E \neq \varnothing$. It suffices to apply Lemma 1.3 to the union $C \cup D$ and obtain a component $K$ of $X \backslash(C \cup D)$ such that $\mathrm{Cl}(K) \cap C \neq \varnothing$ and $\mathrm{Cl}(K) \cap D \neq \varnothing$. Then $E=\mathrm{Cl}(K)$ is a continuum with

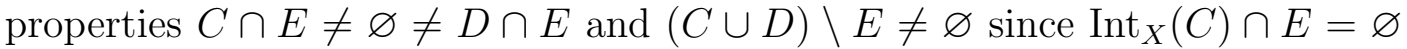
or $\operatorname{Int}_{X}(D) \cap E=\varnothing$.

Step 4. Every restriction

$$
C\left(p_{b}\right) \mid C_{\text {int }}(\lim \mathbf{X}): C_{\text {int }}(\lim \mathbf{X}) \rightarrow C\left(p_{b}\right)\left(C_{\text {int }}(\lim \mathbf{X})\right) \subset C\left(X_{a}\right)
$$

is one-to-one and closed. Consider the inverse system $C(\mathbf{X})=\left\{C\left(X_{a}\right), C\left(p_{a b}\right)\right.$, $A\}$ whose limit is $C(\lim \mathbf{X})$ (Lemma 1.1). From Step 2 it follows that there exists a subset $B$ cofinal in $A$ such that the projections $p_{b}$ are hereditarily irreducible. This means that $C\left(p_{b}\right)$ is light for every $b \in B$ (Proposition 1 ). Since $\lim \mathbf{X}$ is homeomorphic to $\lim \left\{X_{b}, p_{b c}, B\right\}$, we may assume that $B=A$. Let $Y_{a}=C\left(p_{a}\right)\left(C_{\text {int }}(\lim \mathbf{X})\right)$. Furthermore, $C\left(p_{a}\right)^{-1}\left(X_{a}(1)\right)=X(1)$ since from the hereditary irreducibility of $p_{a}$ it follows that no non-degenerate subcontinuum of $X$ maps under $p_{a}$ onto a point. We infer that

$$
\left.C\left(p_{a}\right)^{-1}\left[Y_{a} \backslash X_{a}(1)\right]=C_{i n t}(\lim \mathbf{X})\right) .
$$

Let us prove that the restriction $\left.C\left(p_{a}\right) \mid\left[C_{\text {int }}(\lim \mathbf{X})\right)\right]$ is one-to-one. Suppose that $\left.C\left(p_{a}\right) \mid\left[C_{i n t}(\lim \mathbf{X})\right)\right]$ is not one-to-one. Then there exists a continuum $C_{a}$ (in $X_{a}$ ) and two continua $C, D$ with non-empty interior in $\lim \mathbf{X}$ such that $p_{a}(C)=p_{a}(D)=C_{a}$. It is impossible that $C \subset D$ or $D \subset C$ since $p_{a}$ is hereditarily irreducible. Otherwise, if $C \cap D \neq \varnothing$, then for the continuum $Y=C \cup D$ we would have that $C$ and $D$ are subcontinua of $Y$ and $p_{a}(Y)=$ $p_{a}(C)=p_{a}(D)=C_{a}$, which is impossible since $p_{a}$ is hereditarily irreducible. We infer that $C \cap D=\varnothing$. By Step 3 there exists a continuum $E$ such that $C \cap E \neq$ $\varnothing \neq D \cap E$ and $(C \cup D) \backslash E \neq \varnothing$. Moreover, we may assume that $E \cap C \neq C$ and $E \cap D \neq D$. Now $p_{a}(E \cup D)=p_{a}(E)$, which is impossible since $p_{a}$ is hereditarily irreducible. It follows that the restriction $P_{a}=C\left(p_{a}\right) \mid\left[C_{\text {int }}(\lim \mathbf{X})\right]$ is one-to-one and closed [2, Proposition 2.1.4, p. 95]. 
Step 5. $\left.C_{\text {int }}(\lim \mathbf{X})\right)$ is metrizable and $\mathrm{w}\left(C_{\text {int }}(\lim \mathbf{X})\right) \leq \aleph_{0}$. From Step 4 it follows that $P_{a}$ is a homeomorphism and $\left.C_{\text {int }}(\lim \mathbf{X})\right)$ is metrizable. Moreover, $\mathrm{w}\left(C_{\text {int }}(\lim \mathbf{X})\right) \leq \aleph_{0}$ since $Y_{a}$ as a compact metrizable space is separable and, consequently, second-countable [2, p. 320].

By step 5 the proof is completed.

\section{MAIN THEOREMS}

It is well known that if $X$ is a metric compact space, then there exists a Whitney map for $2^{X}$ and $C(X)$. On the other hand, if $X$ is non-metrizable, then it admits no Whitney map for $2^{X}[1$, Corollary 2, p. 306]. It is known that there exist non-metrizable continua which admit and ones which do not admit a Whitney map for $C(X)$ [1, p. 306]. The properties of continua which admit a Whitney map are investigated in [5] where an external characterization of such continua is obtained, see Theorem 1.2. The problem of an internal characterization of a continuum $X$ which admits a Whitney map for $C(X)$ (posed in [1, Problem 4, p. 306]) is still open. The papers [6] and [8] contain further investigation of the existence of a Whitney map for some special continua. Finally, the paper [7] contains a very general result which states that a D-continuum $X$ admits a Whitney map for $C(X)$ if and only if it is metrizable. Let us note that the class of all D-continua contains semi-aposyndetic continua (in particular, locally connected continua) and arcwise connected continua.

In this section we will consider the class of nearly locally connected continua which are not D-continua and we will prove that every continuum $X$ in this class admits a Whitney map for $C(X)$ if and only if it is metrizable.

A continuum $X$ is said to be near locally connected at a point $x \in X$ provided for every open set $U$ containing $x$ there is a continuum $C$ such that $x \in C \subset U$ and $\operatorname{Int}(C) \neq \varnothing$. A continuum is said to be a $N L C$-continuum provided it is near locally connected at each of its point. Every locally connected continuum is a NLC-continuum.

The concept of aposyndesis was introduced by Jones in [4]. A continuum is said to be semi-aposyndetic [3, Definition 29.1, p. 238], if for every $p \neq q$ in $X$, there exists a subcontinuum $M$ of $X$ such that $\operatorname{Int}_{X}(M)$ contains one of the points $p, q$ and $X \backslash M$ contains the other one. Every locally connected continuum is semi-aposyndetic.

A continuum $X$ is called a $D$-continuum if for any pair $C, D$ of its disjoint non-degenerate subcontinua there exists a subcontinuum $E \subset X$ such that $C \cap E \neq \varnothing \neq D \cap E$ and $(C \cup D) \backslash E \neq \varnothing$.

If $X$ is a locally connected continuum, then $X$ is a D-continuum [8, Lemma 2.4 , p. 150]. Every arcwise connected continuum is a D-continuum.

Example. There exists a non-locally connected non-semi-aposyndetic NLCcontinuum $X$. Moreover, $X$ is not a D-continuum. Let $\mathbb{R}^{2}$ be the Euclidean plane endowed with the ordinary rectangular coordinate system $O x y$. We define the continuum $X$ as a subcontinuum of $\mathbb{R}^{2}$ which is the union of the following sets: 
a) $[-1,0] \times[-1,1]$,

b) $\left\{\left(x, \sin \frac{1}{x}\right): 0<x \leq 1\right\}$,

c) $\left\{\left(x, \sin \frac{1}{2-x}\right): 1 \leq x<2\right\}$,

d) $[2,3] \times[-1,1]$.

It is clear that $X$ is not locally connected. Also, it is not semi-aposyndetic. Namely, if $\left(0, \frac{1}{3}\right)$ and $\left(0, \frac{1}{2}\right)$ are two points of $X$, then each continuum with nonempty interior containing $\left(0, \frac{1}{3}\right)$ contains also $\left(0, \frac{1}{2}\right)$. It is clear that $X$ is locally connected at each point of $X \backslash(\{0\} \times[-1,1] \cup\{2\} \times[-1,1])$. Hence, $X$ is a NLC-continuum at each point of $X \backslash(\{0\} \times[-1,1] \cup\{2\} \times[-1,1])$. On the other hand, for every point $A$ of $\{0\} \times[-1,1] \cup\{2\} \times[-1,1]$ and for every open set containing $A$ there exists a continuum $K$ containing $A$ such that $\operatorname{Int}(K) \neq \varnothing$. Hence $X$ is a NLC-continuum.

Theorem 3.1. Let $X$ be a $N L C$-continuum. Then $\mathrm{w}\left(C_{\text {int }}(X)\right)=\aleph_{0}$ if and only if $\mathrm{w}(X)=\aleph_{0}$.

Proof. If $\mathrm{w}(X)=\aleph_{0}$, then $\mathrm{w}(C(X))=\aleph_{0}$. Hence, $\mathrm{w}\left(C_{i n t}(X)\right)=\aleph_{0}$. Conversely, if $\mathrm{w}\left(C_{\text {int }}(X)\right)=\aleph_{0}$, then there exists a countable base $\mathcal{B}=\left\{B_{i}: i \in \mathbb{N}\right\}$ of $C_{\text {int }}(X) \backslash X(1)$. For each $B_{i}$ let $C_{i}=\cup\left\{x \in X: x \in B, B \in B_{i}\right\}$, i.e., the union of all continua $B$ contained in $B_{i}$.

Claim 1. The family $\left\{C_{i}: i \in N\right\}$ is a network of $X$. Let $X$ be a point of $X$ and let $U$ be an open subset of $X$ such that $x \in U$. There exists and open set $V$ such that $x \in V \subset \mathrm{Cl}(V) \subset U$. Let $K$ be a component of $\mathrm{Cl}(V)$ containing $x$. By the Boundary Bumping Theorem [11, Theorem 5.4, p. 73] $K$ is non-degenerate and, consequently, $K \in C_{i n t}(X)$ since $X$ is an NLC-continuum. Now, $\langle U\rangle \cap\left(C_{\text {int }}(X)\right)$ is a neighbourhood of $K$ in $C_{\text {int }}(X)$. It follows that there exists $B_{i} \in \mathcal{B}$ such that $K \in B_{i} \subset\langle U\rangle \cap\left(C_{\text {int }}(X)\right)$. It is clear that $C_{i} \subset U$ and $x \in C_{i}$ since $x \in K \subset U$. Hence the family $\left\{C_{i}: i \in N\right\}$ is a network of $X$.

Claim 2. $\operatorname{nw}(X)=\aleph_{0}$. Apply Claim 1 and the fact that $\mathcal{B}$ is countable.

Claim 3. $\mathrm{w}(X)=\aleph_{0}$. By Claim 1 we have $\mathrm{nw}(X)=\aleph_{0}$. Moreover, by Theorem $1.4 \mathrm{w}(X)=\aleph_{0}$.

Now we will prove the main result of this paper.

Theorem 3.2. An NLC-continuum $X$ admits a Whitney map for $C(X)$ if and only if it is metrizable.

Proof. By Theorem 2.3 if $X$ is a continuum which admits a Whitney map for $C(X)$, then $C_{\text {int }}(X)$ is a separable metric space. Now, by Theorem $3.1, X$ is metrizable since $X$ is an NLC-continuum.

From the fact that every locally connected continuum is an NLC-continuum and Theorem 3.2 we obtain the following result.

Corollary 3.3. A locally connected continuum $X$ admits a Whitney map for $C(X)$ if and only if it is metrizable.

It is known [2, Corollary 3.1.20, p. 171] that if a compact space $X$ is the countable union of its subspaces $X_{n}, n \in \mathbb{N}$, such that $\mathrm{w}\left(X_{n}\right) \leq \aleph_{0}$, then $\mathrm{w}(X) \leq \aleph_{0}$. Using this fact and Theorem 3.2 we obtain the following theorem. 
Theorem 3.4. Let a continuum $X$ be the countable union of its NLC-subcontinua. Then $X$ admits a Whitney map for $C(X)$ if and only if it is metrizable.

Let $X$ be a topological space. An arc-component of $X$ is a maximal arcwise connected subset of $X$. An arcwise connected continuum contains only one arc-component. Some arc-components are points (degenerate arc-components).

Theorem 3.5. Let $X$ be a continuum which is the countable union of its a-triodic hereditarily unicoherent arc-components. Then $X$ admits a Whitney map for $C(X)$ if and only if it is metrizable.

Proof. Let $\mu$ be a Whitney map for $C(X)$. We will prove that each nondegenerate arc-component of $X$ is the countable union of metrizable arcs. Let $C$ be a non-degenerate arc-component of $X$. Let $a_{1}, b_{1}$ be a pair of distinct points of $C$. There exists an arc $L_{1}=\left[a_{1}, b_{1}\right]$. If $C=\left[a_{1}, b_{1}\right]$, then the proof is completed. Suppose that $C \neq L_{1}$. Let $c$ be any point of $\left[a_{1}, b_{1}\right]$ such that $c \notin\left\{a_{1}, b_{1}\right\}$. Let us recall that $C$ is hereditarily unicoherent and a-triodic. This means that for each arc $[c, x]$ we have the following cases:

1. $[c, x] \subset\left[c, b_{1}\right]$,

2. $[c, x] \supset\left[c, b_{1}\right]$,

3. $[c, x] \subset\left[c, a_{1}\right]$,

4. $[c, x] \supset\left[c, a_{1}\right]$.

Hence we may define two families of arcs. Let $\mathcal{A}$ be a family of all $\operatorname{arcs}[c, x]$ such that either $[c, x] \subset\left[c, a_{1}\right]$ or $[c, x] \supset\left[c, a_{1}\right]$. Similarly, let $\mathcal{B}$ be a family of all arcs $[c, x]$ such that either $[c, x] \subset\left[c, b_{1}\right]$ or $[c, x] \supset\left[c, b_{1}\right]$. If $L_{1}, L_{2}$ is a pair of arcs from $\mathcal{A}(\mathcal{B})$, then either $L_{1} \subseteq L_{2}$ or $L_{1} \supseteq L_{2}$ since $X$ is hereditarily unicoherent an a-triodic. Moreover, if $L_{1}$ is an arc in $\mathcal{A}$ and $L_{2}$ in $\mathcal{B}$, then $L_{1} \cap L_{2}=\{c\}$. Consider a strictly increasing transfinite sequence

$$
\left[c, a_{1}\right] \subset\left[c, a_{2}\right] \subset \cdots \subset\left[c, a_{\alpha}\right], \ldots
$$

of $\operatorname{arcs}$ in $\mathcal{A}$. We may assume that this transfinite sequence is cofinal in $\mathcal{A}$. We will prove that this transfinite increasing sequence is countable. In the opposite case we have an increasing transfinite sequence of real numbers $\mu\left(\left[c, a_{1}\right]\right)<$ $\mu\left(\left[c, a_{2}\right]\right)<\cdots<\mu\left(\left[c, a_{\alpha}\right]\right)<c \ldots, \alpha<\omega_{1}$. This is impossible since $\mathrm{w}(\mathbb{R})=\aleph_{0}$. Similarly, each strictly increasing transfinite sequence

$$
\left[c, b_{1}\right] \subset\left[c, b_{2}\right] \subset \cdots \subset\left[c, b_{\alpha}\right], \ldots
$$

of $\operatorname{arcs} \mathcal{B}$ is countable. This means that we may define a countable family of $\operatorname{arcs}\left\{L_{n}=\left[a_{n}, b_{n}\right]: n \in \mathbb{N}\right\}$. It is clear that $C=\cup\left\{L_{n}: n \in \mathbb{N}\right\}$, i.e., $C$ is the countable union of arcs.

Let $\left\{C_{i}: i \in \mathbb{N}\right\}$ be a family of all arc-components of $X$. Now, $X$ is the countable union of metrizable arcs since every $C_{i}$ is the countable union of metrizable arcs. By virtue of Theorem 3.4, it follows that $X$ is metrizable.

A chain $\left\{U_{1}, \ldots, U_{n}\right\}$ is a finite collection of sets $U_{i}$ such that $U_{i} \cap U_{j} \neq \varnothing$ if and only if $|i-j| \leq 1$. A continuum $\mathrm{X}$ is said to be chainable or arc-like if 
each open covering of $X$ can be refined by an open covering $u=\left\{U_{1}, \ldots, U_{n}\right\}$ such that $\left\{U_{1}, \ldots, U_{n}\right\}$ is a chain.

Corollary 3.6. A chainable continuum $X$ which is the union of countably many arc-components admits a Whitney map for $C(X)$ if and only if it is metrizable.

Proof. Every chainable continuum is hereditarily unicoherent [11, Theorem 12.2, p. 230] and a-triodic [11, Theorem 12.4, p. 231]. So, we can apply Theorem 3.5.

A continuum $X$ is said to be a $\sigma$-rim-nlc continuum provided for every point $x \in X$ and every open set $U$ which contains $x$ there exists an open set $V$ such that $x \in V \subset U$ and the boundary $\operatorname{Bd}(V)$ is the countable union of NLCcontinua.

Theorem 3.7. A $\sigma$-rim-nlc continuum $X$ admits a Whitney map for $C(X)$ if and only if it is metrizable.

Proof. It is known that if $X$ is metrizable, then it admits a Whitney map for $C(X)$ [10, pp. 24-26], [3, p. 106]. Conversely, let $X$ be a $\sigma$-rim-NLC continuum which admits a Whitney map for $C(X)$. We will prove that $X$ is rim-metrizable. Let $x \in X$ be a point of $X$ and let $U$ be an open set containing $x$. There exists an open set $V$ such that $x \in V \subset U$ and the boundary $\operatorname{Bd}(V)=\cup\left\{C_{i}: i \in \mathbb{N}\right\}$ of NLC-continua $C_{i}$. If $\mu: C(X) \rightarrow[0, \infty)$ is a Whitney map, then the restriction $\mu \mid C\left(C_{i}\right)$ is a Whitney map. From Theorem 3.2 it follows that every $C_{i}$ is metrizable since every $C_{i}$ is a NLC-continuum. Using [2, Corollary 3.1.20, p. 171] we conclude that $\operatorname{Bd}(U)$ is metrizable. Finally, from [5, Theorem 11, p. 5] it follows that $X$ is metrizable.

\section{ACKNOWLEDGEMENT}

The author is very grateful to the referee for his/her help and valuable suggestions.

\section{REFERENCES}

1. J. J. Charatonik and W. J. Charatonik, Whitney maps - a non-metric case. Colloq. Math. 83(2000), No. 2, 305-307.

2. R. Engelking, General topology. (Translated from the Polish) Monografie Matematyczne, Tom 60. [Mathematical Monographs, Vol. 60]. PWN-Polish Scientific Publishers, Warsaw, 1977.

3. A. Illanes and S. B. Nadler, JR., Hyperspaces. Fundamentals and recent advances. Monographs and Textbooks in Pure and Applied Mathematics, 216. Marcel Dekker, Inc., New York, 1999.

4. F. B. Jones, Aposyndetic continua and certain boundary problems. Amer. J. Math. 63(1941), 545-553.

5. I. LonČAR, A note on a Whitney map for continua. Math. Commun. 6(2001), No. 1, 1-9.

6. I. LonČAR, A fan $X$ admits a Whitney map for $C(X)$ iff it is metrizable. Glas. Mat. Ser. III 38(58)(2003), No. 2, 395-411. 
7. I. LONČAR, D-continuum $X$ admits a Whitney map for $C(X)$ if and only if it is metrizable. Glas. Mat. Ser. III 40(60)(2005), No. 2, 333-337.

8. I. LONČAR, Whitney map for hyperspaces of continua with the property of Kelley. JP J. Geom. Topol. 4(2004), No. 2, 147-156.

9. E. Michael, Topologies on spaces of subsets. Trans. Amer. Math. Soc. 71(1951), 152182.

10. S. B. NADler, JR., Hyperspaces of sets. A text with research questions. Monographs and Textbooks in Pure and Applied Mathematics, Vol. 49. Marcel Dekker, Inc., New YorkBasel, 1978.

11. S. B. NAdLer, JR., Continuum theory. An introduction. Monographs and Textbooks in Pure and Applied Mathematics, 158. Marcel Dekker, Inc., New York, 1992.

12. J. Nikiel, H. M. Tuncali, and E. D. Tymchatyn, Continuous images of arcs and inverse limit methods. Mem. Amer. Math. Soc. 104(1993), No. 498, viii+80 pp.

13. G. T. Whyburn, Analytic topology. American Mathematical Society Colloquium Publications, Vol. XXVIII. American Mathematical Society, Providence, R.I., 1963.

(Received 16.03.2006)

Author's address:

Faculty of Organizations and Informatics Varaždin

Croatia

E-mails: ivan.loncar1@vz.htnet.hr

ivan.loncar@foi.hr 